\title{
Cardiovascular Events in Hemodialysis Patients: Challenging against Vascular Calcification
}

\author{
Shuzo Kobayashi, MD, PhD
}

Particularly in patients with hemodialysis, cardiovascular mortality rate is extremely high. Polyvascular diseases develop at an early stage of chronic kidney disease (CKD). Pathophysiology includes insulin resistance and/or imbalance between nitric oxide (NO) and endothelin bioavailability as well as oxidative stress. The understanding in pathophysiology of vascular calcification and strategic treatment is a critical issue to achieve favorable outcome for the patients with CKD. In this article, we aim to review the cardiovascular disease for the patients with CKD with a particular emphasis on the clinical aspects of polyvascular disease. Finally, we address to detect microcirculatory impairment and eradicate vascular calcification as early as possible prior to renal replacement therapy. (This is a translation of Jpn J Vasc Surg 2016; 25: 359-365.)

Keywords: cardiovascular events, PAD, coronary, hemodialysis

\section{Introduction}

In patients undergoing dialysis, cardiovascular lesions may appear in the early stage of pre-dialysis chronic kidney disease (CKD), stage $3 \mathrm{~b}$ (GFR: $<45 \mathrm{~mL} / \mathrm{min} / 1.73 \mathrm{~m}^{2}$ ). Coronary calcification, as an alternative marker, becomes marked in this stage (Fig. 1). Insulin resistance and asymmetric dimethylarginine (ADMA), which inhibits nitric oxide (NO) synthase, are involved in the pathogenesis. ${ }^{1,2)}$ A high level of insulin in blood promotes the proliferation of vascular smooth muscle cells, increasing sympathetic nerve activity, activating the renin-angiotensin system, and increasing $\mathrm{Na}$ resorption in the kidney tubule. Various factors in the presence of CKD are involved in insulin resistance (Table 1), as described below, and they appear in the early stage of CKD.

Thus, CKD is an independent risk factor for cardiovascular disease. ${ }^{3)}$ A study reported that many patients died of

Hemodialysis Center, Shonan Kamakura General Hospital, Kamakura, Kanagawa, Japan

Received: January 19, 2017; Accepted: January 20, 2017 Corresponding author: Shuzo Kobayashi, MD, PhD. Hemodialysis Center, Shonan Kamakura General Hospital, 1370-1 Okamoto, Kamakura, Kanagawa 247-8533, Japan

Tel: +81-467-46-1717, Fax: +81-467-45-0190

E-mail: shuzo@shonankamakura.or.jp

This is a translation of Jpn J Vasc Surg 2016; 25: 359-365. cardiovascular disease before the introduction of dialysis. ${ }^{4)}$

On the other hand, vascular lesions may develop/become advanced before the introduction of dialysis, but, actually, vascular calcification may further progress after introduction. Stenosis of the aortic valve or lower limb peripheral artery lesions are difficult to treat, raising important issues for dialysis-specializing physicians, vascular surgeons, and cardiologists.

In this article, we review the pathogenesis of cardiovascular lesions, coronary artery disease, peripheral artery disease, and valvular disease, in patients undergoing dialysis.

\section{GFR and Coronary artery calcification (CACS)}

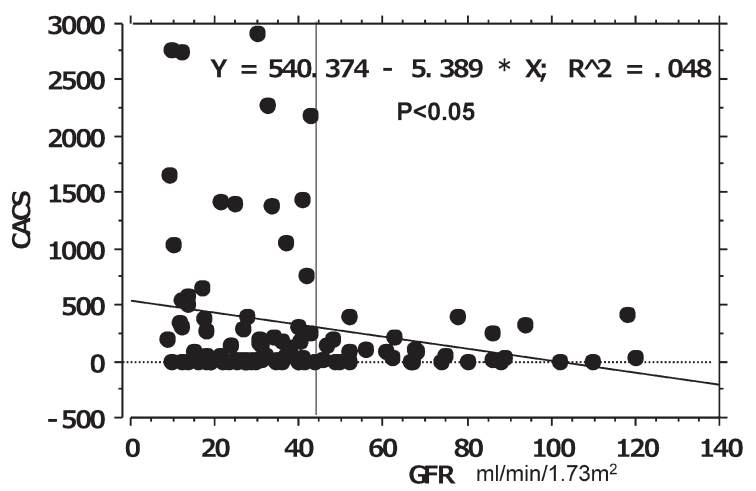

Fig. 1 Coronary artery calcification (CACS) develops at an early stage of renal dysfunction.

Table 1 Factors affecting insulin resistance
1. Anemia
2. $\mathrm{PTH}$
3. 1, 25- $(\mathrm{OH})_{2}$ vitamin $\mathrm{D}_{3}$
4. Protein/keto-acid analogue
5. Guanidino substrates
6. Exercise
7. Acidemia
8. Dyslipidemia
9. Chronic inflammation (adipocytokine, adiponectin)
10. Aldosterone \& Angiotensin II
11. FGF-23
12. Gastrointesitinal polypeptide (Ghrelin)
13. Others (Uremic toxin, $p$-Cresyl sulfate) 


\section{Vascular Calcification-Influence of Drugs (Warfarin, Ca-containing Phosphorus Ad- sorbents)}

Clinical factors for vascular calcification are presented in Table 2. Vascular calcification is not solely associated with the passive binding of phosphorus to calcium. Concerning its mechanism, bone-associated proteins, such as FetuinMatrixGla protein (MGP) and osteoprotegerin, may be involved in the transformation of vascular smooth muscle cells to osteoblasts (Table 2). In particular, MGP inhibits topical calcification, and the use of Warfarin, which reduces MGP, promotes vascular calcification. In the guidelines for cardiovascular disease established by the Japanese Society for Dialysis Therapy, the limited use of Warfarin is recommended.

However, the most potent calcification-promoting factor is a phosphorus adsorbent containing $\mathrm{Ca}$ (calcium carbonate). It is used to decrease the blood level of phosphorus, which contributes to calcification, but it may promote calcification. Recently, Ca-free phosphorus adsorbents have been introduced, and they may exhibit inhibitory effects on calcification in the future. Previously, we conducted a prospective interventional study using lanthanum carbonate, and reported that coronary artery calcification was inhibited. ${ }^{5)}$

Uremia plays an important role in the pathogenesis of innate immunity to inflammation. The involvement of uremic toxin remains to be clarified. Etiological factors

Table 2 Factors related with vascular calcifications

\begin{tabular}{l} 
Induction \\
Age \\
Phosphorus \\
Inflammation \\
AGE \\
Klotho/FGF-23 \\
BMP2/4 \\
Leptin \\
Collagen/Fibronectin \\
LDL-ox \\
Inhibition \\
Adiponectin \\
BMP7 \\
Fetuin \\
Osteoprotegerin \\
PTHrP \\
Osteopontin \\
Pyrophosphate \\
Collagen IV \\
MGP \\
\hline
\end{tabular}

AGE: advanced glycation endoproduct; BMP: bone morphogenic protein; LDL-ox: oxidized low density lipoprotein; PTHrP: PTHrelated protein include a GFR of $<45 \mathrm{~mL} / \mathrm{min}$, especially $<30 \mathrm{~mL} / \mathrm{min}$. In many cases, the blood phosphorus level increases immediately before renal replacement therapy is required due to advanced renal failure (GFR: $\leq 20 \mathrm{~mL} / \mathrm{min}$ ). However, prior to this finding, there is an increase in the parathyroid hormone (PTH) level and a decrease in the active vitamin D3 level. Recently, the timing and pathogenesis of inflammation have been clarified ${ }^{6}$ : before the above changes, there is an increase in the FGF-23 level and a reduction in the expression of Klotho gene, which will be examined as an important factor in the future. Such a pathogenesis, including acidemia, other than classical risk factors, such as blood pressure and dyslipidemia, must be carefully evaluated in the process of pre-dialysis kidney disease progression and managed in the early stage.

\section{Insulin Resistance, Vascular Endothelial Disorder, and Abnormalities in Rheology}

Vascular lesions show pathological findings including atherosclerosis in vascular endothelial cells and Monckeberg's medial calcific sclerosis, ${ }^{7)}$ which was proposed by a pathologist (Germany). Endothelial disorder is associated with insulin resistance, and platelets are activated through adhesion/aggregation. In patients undergoing dialysis, a large number of monocytes and platelets are aggregated. ${ }^{8)}$ In particular, in such patients, the number of monocyte/ platelet aggregates further increases in the presence of peripheral artery disease; hemorheology may be affected. Considering apparently contradictory conditions, such as the bleeding tendency related to platelet dysfunction and hypercoagulability associated with a high blood level of fibrinogen, the use of anti-platelet drugs remains controversial. A study reported that insulin resistance appeared in the early stage of pre-dialysis renal hypofunction, sug-

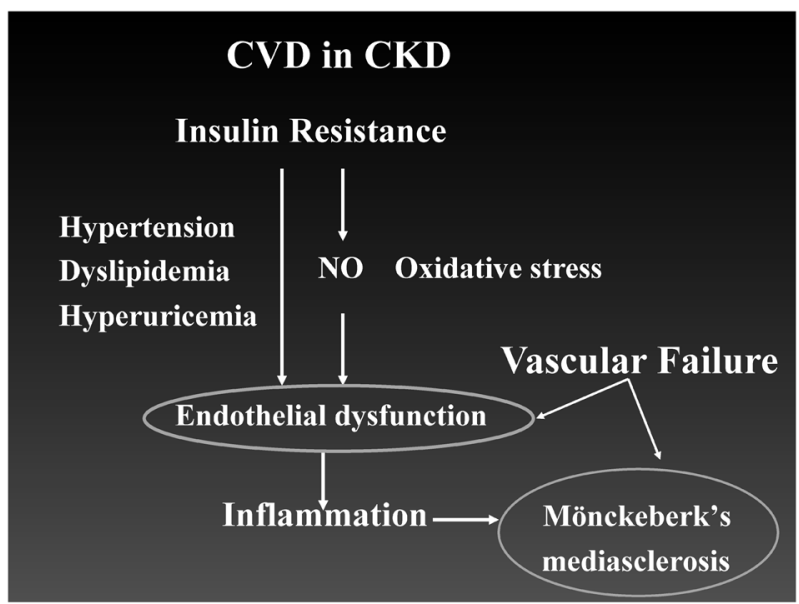

Fig. 2 Cardiovascular disorders are related with insulin resistance, thus leading to endothelial dysfunction. 
gesting the involvement of acidemia or dyslipidemia. ${ }^{1)}$ Disturbance of the endothelium/media may lead to vascular failure (Fig. 2).

\section{Polyvascular Disease - With Respect to Pe- ripheral Microcirculatory Impairment-}

The entity of polyvascular disease was proposed by the Reduction of Atherothrombosis for Continued Health (REACH) Registry.9)

This was a prospective, cross-sectional, observational study of atherothrombotic disease, of which the incidence has increased, involving outpatients in various countries. The disease refers to atherothrombosis with 2 or more symptoms at the coronary/cerebral/lower limb peripheral arterial bed or circulatory bed. As CKD is an independent risk factor for cardiovascular disorder, ${ }^{3)}$ polyvascular disease must be considered for diagnosis/treatment when 1 cardiovascular disorder is detected.

However, early coronary/peripheral artery abnormalities may be derived from abnormalities in rheology, as described above, or peripheral microcirculatory disorder. It is necessary to detect metabolic myocardial abnormalities associated with ischemia at the capillary level around the cardiac muscle in the early phase using BMIPP scintigraphy before coronary angiography. In addition, using echocardiography, the deterioration of left ventricular hypertrophy should be detected in the early stage.

\section{Coronary Artery Disease in Patients Under- going Dialysis}

As described above, calcification of the coronary artery is an independent relevant factor for cardiovascular events or cardiovascular death in CKD patients. Its incidence was significantly higher in those with stage G3 or higher CKD, and coronary artery calcification may contribute to left ventricular hypertrophy or a reduction in coronary blood flow. ${ }^{2)}$ As a result, the overall mortality rate and incidence of cardiovascular events significantly increased with a decrease in the glomerular filtration rate (GFR) before the introduction of dialysis. ${ }^{4)}$ Of patients without cardiac abnormalities at the time of dialysis introduction, coronary angiography revealed stenosis of the coronary artery in $50 \%$, which shocked many health care professionals. Some patients had 2-/3-vessel lesions, and others required percutaneous coronary intervention (PCI). Of patients with diabetes, coronary stenosis was observed in $89 \% .^{10)}$ Before coronary angiography, it is necessary to detect abnormalities in the phase of capillary-myocyte mismatch in capillary microcirculation around cardiomyocytes.

\section{Peripheral Artery Disease in Patients Un- dergoing Dialysis}

Peripheral artery disease (PAD) of the lower limbs in patients undergoing dialysis is characterized by a high morbidity rate, difficulty in treatment related to vascular calcification and peripheral lesion sites of the lower limbs, a high mortality rate related to lower-limb amputation, and rapid ulcer appearance after the asymptomatic phase. ${ }^{11,12)}$

Therefore, early detection in the asymptomatic phase is important.

The lower-limb amputation rate in patients undergoing dialysis has annually increased. At the end of 2000, it was $1.6 \%$, but it increased to $2.6 \%(4,755 / 240,000$ persons) at the end of 2005 . Of these, $70 \%$ had diabetes. In 2014, lower-limb-amputated patients accounted for $3.7 \%$. During the past 10 years, the number of lowerlimb-amputated patients in those undergoing dialysis has increased $\geq 2$-fold (approximately 4,000 $\rightarrow 9,000$ ). Furthermore, the prognosis after lower-limb amputation is extremely unfavorable, and the 1-year mortality rate is approximately $50 \%$. The frequent causes of death in PAD patients include cardio-/cerebrovascular disorder, malnutrition, and infection at necrotic ulcer sites. PAD, as a primary disease involved in mortality, may be overlooked, but the prognosis after lower-limb amputation in dialysis patients is markedly poorer than in patients with myocardial infarction. ${ }^{12,13)}$ For early detection, it is important to evaluate peripheral microcirculatory disorder using the toe-brachial pressure index (TBI) or skin perfusion pressure (SPP), as the sensitivity of the ankle-brachial pressure index (ABI) is low due to vascular calcification. ${ }^{14)}$ If a diagnosis is made based on the ABI alone, the sensitivity is markedly low. When the ABI is 0.9 , the specificity is $100 \%$, whereas the sensitivity is $30 \%$. Considering this, the specificity and sensitivity of an SPP of $50 \mathrm{mmHg}$ are 77 and $85 \%$, respectively; this parameter is appropriate for screening. For treatment, positive revascularization is essential, but multidisciplinary treatment involving infection control, nutrition, and rehabilitation should be performed.

In dialysis patients, treatment is difficult if an ulcer appears, and the prognosis after lower-limb amputation is markedly poor. Therefore, early diagnosis and treatment, involving adequate foot care, are important. ${ }^{12)}$

PAD is frequently observed after the introduction of dialysis, as indicated for coronary artery disease. Based on the results of evaluation using the ABI and SPP, approximately $25 \%$ of dialysis-introduction-phase patients and approximately $40 \%$ of those undergoing maintenance dialysis had PAD. In dialysis patients with $\mathrm{PAD}$, the incidence of cardio-/cerebrovascular disorder is markedly higher than in those without PAD.

A study examined the lower-limb arterial calcification 
score in dialysis patients, and reported that the grade of such calcification was directly associated with the severity of PAD. ${ }^{15)}$ Lower-limb arterial calcification is diffusely observed in the lower limb arteries above/below the knees, reducing the accuracy of the ABI for PAD diagnosis and making the CT-based assessment of vascular stenosis or revascularization difficult. In particular, a 3-year prospective observational study showed that the onset of ulcers led to lower-limb amputation in approximately $15 \%$ of Fontaine grade I patients undergoing dialysis. This suggests that the absence of symptoms does not always reflect the absence of ischemia; the adequate use of anti-platelet drugs in the early stage should be considered/reviewed, as described above.

PAD induces vascular calcification, as demonstrated for coronary artery disease.

In dialysis patients, critical limb ischemia (CLI) with multiple severe lesions of 3 peripheral artery vessels below the knees (anterior/posterior tibial and peroneal arteries) is frequently observed. In this case, it is difficult to save the limbs by treating the iliac or superficial femoral arteries alone; revascularization in these peripheral artery regions is required. Lower thigh artery treatment has been difficult, but, recently, positive endovascular treatment using devices for PCI has been increasingly performed. This treatment is minimally invasive, and it can be repeatedly conducted, with a low incidence of complications; therefore, it may be indicated for a larger number of patients in the future. However, the restenosis rate is high $(\geq 50 \%)$, and the management of severe lesions at the pedal or plantar arches, especially calcification, is limited, raising future issues. Furthermore, endovascular treatment for significantly stenotic lesions in the absence of CLI should be reviewed.

Based on the results of some studies after the BASIL study, ${ }^{16,17)}$ endovascular therapy (EVT) is used as firstline treatment due to its low-level invasiveness. However, bypass is recommended, considering the prognosis 2 years or more after EVT.

Concerning anti-platelet drugs, the platelet function is affected in patients with CKD, as described above. On the other hand, there is an inflammation-related increase in the blood fibrinogen level, or heparin is used at each time of dialysis; it is difficult to indicate anti-platelet drugs. However, several studies using the SPP showed that the use of anti-platelet drugs markedly improved peripheral microcirculatory disorder. ${ }^{18,19)}$

\section{Stenosis of the Aortic Valve in Dialysis Patients}

Calcification is frequently observed. A study reported that there was a negative correlation between calcification and the concentration of bicarbonate before dialysis. ${ }^{20)}$ Sufficient dialysis or nutritional improvement is important. Acidemia control for dialysis patients, involving the type of dialytic fluid, must be examined.

In the guidelines for cardiovascular disease established by the Japanese Society for Dialysis Therapy, the speed of progression is emphasized. Narrowing of the valve orifice area progresses by $0.23 \mathrm{~cm}^{2} /$ year. Unless surgery is performed at a valve orifice area of $\geq 0.6 \mathrm{~cm}^{2}$, stenosis may rapidly progress, leading to sudden death during dialysis or making management difficult due to dialysis-related hypotension. According to recent studies, if there is a rapid decrease in the blood pressure during dialysis or shock is observed, stenosis of the aortic valve should be differentiated rather than coronary ischemia or severe arrhythmia.

\section{Discussion}

Hyperphosphatemia is a prognostic factor for cardiovascular disorder and the deterioration of renal dysfunction in patients with CKD. In those with a serum phosphorus level of $\geq 4.5 \mathrm{mg} / \mathrm{dL}$, the mortality rate is 2 times higher than in those with a serum phosphorus level of 2 to $3 \mathrm{mg} / \mathrm{dL}$. Therefore, phosphorus control in the pre-dialysis phase of CKD is important. In evidence-based guidelines for the management of CKD in 2013,21) it is recommended that the target of phosphorus control in the pre-dialysis phase should be established as its normal range (2.5 to $4.5 \mathrm{mg} / \mathrm{dL}$ ) regardless of the stage of CKD. For dialysis patients, it is recommended that the serum level of phosphorus should be maintained at 3.5 to $6.0 \mathrm{mg} / \mathrm{dL}$. However, many investigators consider that this range is too high. Even in patients with a phosphorus level within this range, there are patients with a high fibroblast growth factor level. In these patients, the risk of cardiovascular death is markedly high. Therefore, considering the FGF23 level, the optimal level of phosphorus control must be reviewed in the future. It is necessary to select new-type Ca-free phosphorus adsorbents, such as lanthanum- or ironcontaining preparations, and restrict the use of Warfarin (after mechanical valve replacement or secondary prevention of cerebral embolism). The use of new non-vitamin$\mathrm{K}$-dependent oral drugs is also restricted in accordance with the GFR, but the FDA (U.S.A.) approved the use of apixaban for dialysis patients last year. ${ }^{22)}$

On the other hand, dialysis modalities should be examined from various viewpoints: which of two procedures, hemodialysis (HD) and hemodiafiltration (HDF), should be selected? If the former is selected, which dialyzer should be used? If the latter is selected, which displacement liquid volume, 10 or $40 \mathrm{~L}$, should be adopted? Is acetate-free dialytic fluid appropriate? What is the optimal Ca concentration? A study suggested the inhibitory effects of 
vitamin-E-fixed dialyzers on arteriosclerosis. ${ }^{23)}$ In addition, another study indicated that online HDF pre-dilution replacement at $40 \mathrm{~L}$ improved aorta stiffness or the left ventricular myocardial weight index (LVMI). ${ }^{24)}$

\section{Strategies to Prevent Cardiovascular Disorder}

Various drug therapies are recommended to prevent cardiovascular disorder in CKD patients. Preparations other than the phosphorus adsorbents described above are presented below.

\section{RAS inhibitors (ACE inhibitors/ARB)}

Many studies reported the reno- and cardioprotective effects of RAS inhibitors. Combination therapy with an ACE inhibitor and ARB is more effective for proteinuria than monotherapy with respective drugs, but no study has reported its usefulness with respect to death or creatinine doubling; some studies indicated deterioration, and it is not recommended.

\section{Statins}

A large-scale randomized double-blind trial (SHARP study) investigated whether lipid intervention for CKD patients inhibits arteriosclerotic events; 9,270 CKD patients with a mean eGFR of $26.6 \mathrm{~mL} / \mathrm{min} / 1.73 \mathrm{~m}^{2}$ were assigned to receive combination therapy with simvastatin at $20 \mathrm{mg} /$ day and ezetimibe at $10 \mathrm{mg} /$ day or a placebo, and followed-up (mean follow-up period: 4.9 years). In the therapeutic intervention group, the risk of first major atherosclerotic events (nonfatal myocardial infarction, coronary death, cerebral infarction, coronary revascularization) decreased by $17 \%$. In the KDIGO guidelines and guidelines for the management of CKD in 201321) in Japan, intervention for dyslipidemia in the pre-dialysis phase of CKD is recommended from the viewpoint of cardiovascular disorder prevention.

\section{Vitamin D}

A study reported that the oral administration of a lowdose vitamin $\mathrm{D}$ preparation ( $1 \alpha$ hydroxyvitamin $\mathrm{D}_{3}$ ) significantly decreased the risk of cardiovascular death in dialysis patients. Low-dose vitamin D administration, which does not influence vascular calcification related to an increase in the calcium/phosphorus product, may be useful in dialysis patients.

A review of all stages of CKD (13 randomized controlled trials (RCTs), including 5 involving dialysis patients) indicated that there were no significant improvements in the cardiovascular or overall mortality rates. However, in these RCTs, the sample size was small, and the risk of death was not sufficiently examined (an improvement in the PTH level or changes in the LVMI were established as a primary endpoint). In addition, the dose of vitamin $\mathrm{D}$ and administration period varied among the trials; currently, there is no evidence regarding the effects of administering vitamin $\mathrm{D}$ preparations to CKD patients. However, the results of a prospective interventional study will be published soon (J-DAVID Study).

\section{Aspirin}

Aspirin is recommended for the prevention of cardiovascular death in high-risk patients with a history of myocardial or cerebral infarction and secondary prevention of vascular disorder. In CKD patients, the incidence of cardiovascular disorder is particularly high, and many patients have been taking aspirin. However, the data on the usefulness of aspirin administration to CKD patients is limited.

A study (non-RCT) compared 1,884 CKD patients receiving aspirin with the same number of non-aspirintreated patients with CKD by propensity score matching, and reported that the incidence of cardiovascular events was significantly higher in the former, and that the creatinine doubling and renal mortality rates were significantly higher. ${ }^{25)}$ There were no differences in the overall mortality rate or incidence of hemorrhagic events between the two groups. However, the results suggest that the use of aspirin in CKD patients is harmful with respect to cardiovascular disorder or renal dysfunction.

\section{Drugs for hyperuricemia}

A drug for hyperuricemia, allopurinol, was confirmed to inhibit the deterioration of renal hypofunction and decrease the incidence of cardiovascular events by $71 \%$. Febuxostat, which was recently introduced, exhibits similar inhibitory effects on cardiovascular disorder without inducing adverse reactions.

\section{Erythropoiesis-stimulating agents (ESA)}

Renal anemia treatment with ESA may inhibit the onset of cardiovascular events, but the risk of such events may increase when establishing a target hemoglobin $(\mathrm{Hb})$ level as $\geq 12$ to $13 \mathrm{~g} / \mathrm{dL}$ (CHOIR). ${ }^{26)}$

Thus, the incidence of cardiovascular disorder is high in CKD patients, and this is not limited to dialysis patients. Strategies to prevent cardiovascular complications should be performed in the pre-dialysis phase of CKD. The preventive effects of various drugs on cardiovascular complications have been reported, as described above. These drugs should be adequately combined for pre-dialysis CKD management.

\section{Conclusion}

In dialysis patients, insulin resistance-based cardiovascular 
disorder appears in the early stage of renal hypofunction before the initiation of dialysis. In addition to oxidative stress, organ microcirculatory impairment and vascular calcification play an important role. Such cardiovascular disorder deteriorates after the introduction of dialysis, and phosphorus control, dialyzer selection, and dialysis modalities, such as HDF, are involved in the pathogenesis; therefore, dialysis should be sufficiently conducted.

To evaluate cardiovascular disorder in the early stage, metabolic blood flow scintigraphy or peripheral microcirculatory impairment assessment should be performed in addition to echocardiography or angiography.

Improvements in the prognosis of cardiovascular disorder or treatment results are achieved through fights with vascular calcification. In the future, the grade of vascular calcification may be reduced by minimizing the use of calcium carbonate or Warfarin and improving uremia (inflammation) through sufficient dialysis.

\section{Disclosure Statement}

The author received lecture fees from Bayer Yakuhin, Ltd., Kaneka Corporation, and Chugai Pharmaceutical Co., Ltd. There is no other conflict of interest, involving relatives.

\section{Additional Note}

Year of announcement: May 27, 2016, Tokyo

\section{References}

1) Kobayashi S, Maesato K, Moriya H, et al. Insulin resistance in patients with chronic kidney disease. Am J Kidney Dis 2005; 45: 275-80.

2) Kobayashi S, Oka M, Maesato K, et al. Coronary artery calcification, ADMA, and insulin resistance in CKD patients. Clin J Am Soc Nephrol 2008; 3: 1289-95.

3) Sarnak MJ, Levey AS, Schoolwerth AC, et al. Kidney disease as a risk factor for development of cardiovascular disease: a statement from the American Heart Association Councils on Kidney in Cardiovascular Disease, High Blood Pressure Research, Clinical Cardiology, and Epidemiology and Prevention. Circulation 2003; 108: 2154-69.

4) Go AS, Chertow GM, Fan D, et al. Chronic kidney disease and the risks of death, cardiovascular events, and hospitalization. N Engl J Med 2004; 351: 1296-305.

5) Ohtake T, Kobayashi S, Oka M, et al. Lanthanum carbonate delays the progression of coronary artery calcification compared with calcium-based phosphate binders in paients on hemodialysis: a pilot study. J Cardiovasc Pharmacol Ther 2013; 18: 439-46.

6) Kobayashi S. Cardiovascular events in chronic kidney disease (CKD) - an importance of vascular calcification and microcirculatory impairment. Ren Replace Ther 2016; 2: 55.

7) Drüeke TB. Arterial intima and media calcification: distinct entities with different pathogenesis or all the same? Clin J Am Soc Nephrol 2008; 3: 1583-4.

8) Kobayashi S, Okamoto K, Maesato K, et al. Important role of blood rhology in atherosclerosis of patients with hemodialysis. Hemodial Int 2005; 9: 268-74.

9) Bhatt DL, Steg PG, Ohman EM, et al. International prevalence, recognition, and treatment of cardiovascular risk factors in outpatients with atherothrombosis. JAMA 2006; 295: 180-9.

10) Ohtake T, Kobayashi S, Moriya H, et al. High prevalence of occult coronary artery stenosis in patients with chronic kidney disease at the initiation of renal replacement therapy: an angiographic examination. J Am Soc Nephrol 2005; 16: 1141-8.

11) Kobayashi S. Early detection of lower limbs' ischemia and strategies for the treatment. IGAKU NO AYUMI 2012; 240 : 903-8. (in Japanese)

12) Kobayashi S. CKD and progression of PAD. J Jpn Soc Intern Med 2016; 105: 842-9. (in Japanese)

13) Aulivola B, Hile CN, Hamdan AD, et al. Major lower extremity amputation: outcome of a modern series. Arch Surg 2004; 139: 395-9; discussion, 399.

14) Okamoto K, Oka M, Maesato K, et al. Peripheral arterial occlusive disease is more prevalent in patients with hemodialysis: comparison with the findings of multidetector-row computed tomography. Am J Kidney Dis 2006; 48: 269-76.

15) Ohtake T, Oka M, Ikee R, et al. Impact of lower limbs' arterial calcification on the prevalence and severity of PAD in patients on hemodialysis. J Vasc Surg 2011; 53: 676-83.

16) Bradbury AW, Adam DJ, Bell J, et al. BASIL trial Participants. Bypass versus Angioplasty in Severe Ischaemia of the Leg (BASIL) trial: analysis of amputation free and overall survival by treatment received. J Vasc Surg 2010; 51 Suppl: 18S-31S.

17) Shiraki T, Iida O, Takahara M, et al. Comparison of clinical outcomes after surgical and endovascular revascularization in hemodialysis patients with critical limb ischemia. J Atheroscler Thromb E-pub ahead of print Oct 26, 2016.

18) Hidaka S, Kobayashi S, Iwagami M, et al. Sarpogrelate hydrochloride, a Selective $5-\mathrm{HT}_{2 \mathrm{~A}}$ receptor antagonist, improves skin perfusion pressure of the lower extremities in hemodialysis patients with peripheral arterial disease. Ren Fail 2013; 35: 43-8.

19) Ohtake T, Sato M, Nakazawa R, et al. Randomized pilot trial between prostaglandin $\mathrm{I}_{2}$ analog and anti-platelet drugs on peripheral arterial disease in hemodialysis patients. Ther Apher Dial 2014; 18: 1-8.

20) Oka M, Ohtake T, Mochida Y, et al. Correlation of coronary artery calcification with pre-hemodialysis bicarbonate levels in patients on hemodialysis. Ther Apher Dial 2012; 16: $267-$ 71.

21) Japanese Society of Nephrology ed. Evidence-based Clinical Practice Guideline for CKD. Tokyo: Tokyo Igakusha, 2013. (in Japanese)

22) Chan KE, Giugliano RP, Patel MR, et al. Nonvitamin K anticoagulant agents in patients with advanced chronic kidney disease or on dialysis with AF. J Am Coll Cardiol 2016; 67: 2888-99.

23) Kobayashi S, Moriya H, Aso K, et al. Vitamin E-bonded hemodialyzer improves atherosclerosis associated with a rheo- 
logical improvement of circulating red blood cells. Kidney Int 2003; 63: 1881-7.

24) Ohtake T, Oka M, Ishioka K, et al. Cardiovascular protective effects of online hemodiafiltration: comparison with conventional hemodialysis. Ther Apher Dial 2012; 16: 181-8.

25) Kim AJ, Lim HJ, Ro H, et al. Low-dose aspirin for preven- tion of cardiovascular disease in patients with chronic kidney disease. PLoS One 2014; 9: e104179.

26) Singh AK, Szczech L, Tang KL, et al. Correction of anemia with epoetin alfa in chronic kidney disease. N Engl J Med 2006; 355: 2085-98. 